\title{
Symmetry of the Pyritohedron and Lattices
}

\author{
Nazife O. Koca*, Aida Y. Al-Mukhaini, Mehmet Koca, and Amal J. Al-Qanobi
}

Department of Physics, College of Science, Sultan Qaboos University, P.O. Box 36, PC 123 Al-Khoud, Muscat, Sultanate of Oman. *Email: nazife@squ.edu.om.

\begin{abstract}
The pyritohedron consisting of twelve identical but non regular pentagonal faces and its dual pseudoicosahedron that possess the pyritohedral $\left(T_{h}\right)$ symmetry play an essential role in understanding the crystallographic structures with the pyritohedral symmetry. The pyritohedral symmetry takes a simpler form in terms of quaternionic representation. We discuss the $3 \mathrm{D}$ crystals with the pyritohedral symmetry which can be derived from the Coxeter-Dynkin diagram of $D_{3}$.
\end{abstract}

Keywords: Pseudoicosahedron; Pyritohedron; Lattice; Coxeter groups and Quaternions.

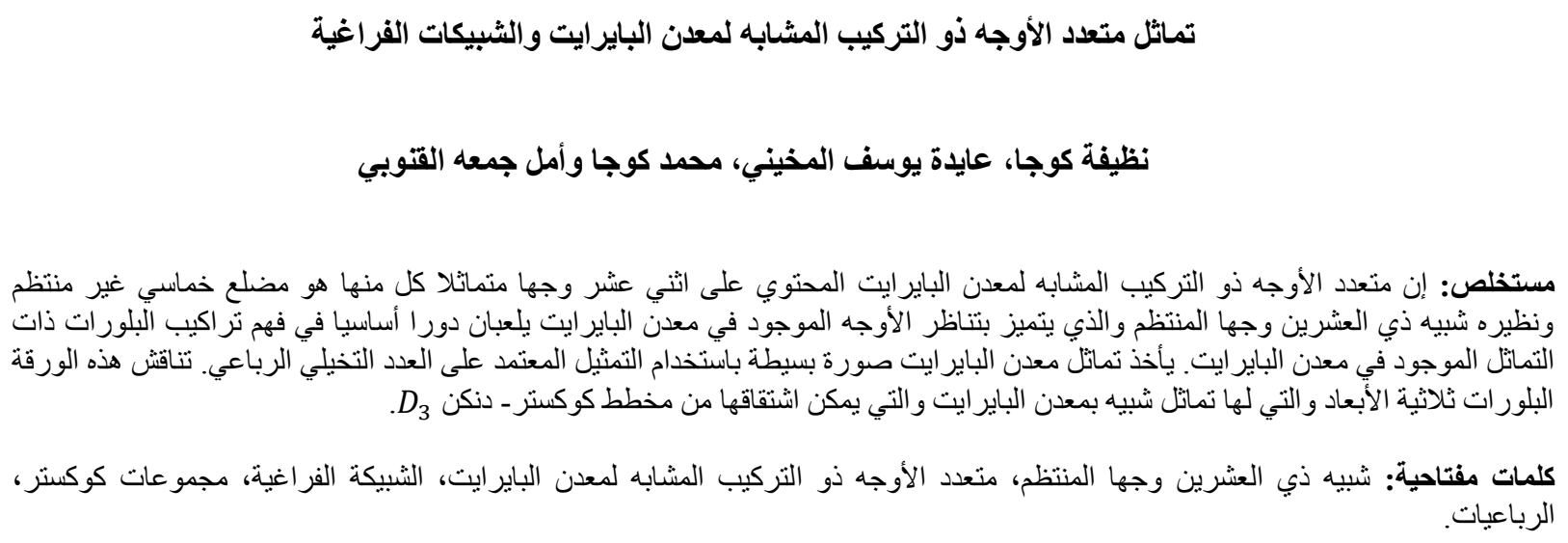

\section{Introduction}

Qymmetry describes the periodic repetition of structural features. Any system exhibits symmetry if the action of the symmetry operations leaves the system apparently unchanged. Crystals possess a regular, repetitive internal structure, therefore they have symmetry. Coxeter groups are the symmetry groups generated by reflections [1]. They describe the symmetry of regular and semi-regular polytopes in arbitrary dimensions [1]. The CoxeterWeyl groups acting as discrete groups in 3D Euclidean space generate orbits representing vertices of certain polyhedra [2-3]. Rank-3 Coxeter-Weyl groups $W\left(D_{3}\right)$ and $W\left(B_{3}\right) \approx \operatorname{Aut}\left(D_{3}\right)$ define the point tetrahedral and octahedral symmetries of the cubic lattices [4]. In this work we use Coxeter-Dynkin diagram $D_{3}$ and construct the pyritohedral group and the related polyhedra in terms of quaternions by finding the vertices of the pseudoicosahedron with pyritohedral symmetry.

We organize the paper as follows. In Section 2 we introduce quaternions and their relevance to $O(3)$ and $O$ (4) transformations. The sets of quaternions defining the binary tetrahedral and binary octahedral groups are given. Section 3 explains Coxeter-Dynkin diagram of $D_{3}$ in which the simple roots (lattice generating vectors) and the group generators are expressed in terms of imaginary quaternionic units and the reflection planes of the diagram $D_{3}$ are identified as certain planes of the unit cube. Section 4 deals with the construction of the icosahedron, dodecahedron, pseudoicosahedron and pyritohedron from the pyritohedral group derived from the $D_{3}$ diagram as its automorphism group. It is noted that the truncated octahedron, the Wigner-Seitz cell of the BCC lattice, splits into two pseudoicosahedra which are mirror images of each other. In Section 5 we discuss the pseudoicosahedron and its dual pyritohedron to give a description of those crystals possessing pyritohedral symmetry. 


\section{Quaternions and their Relevance to the Isometries of the $O(3)$ and $O$ (4) Transformations}

Quaternions are vectors in four dimensions provided with a rule for multiplication that is associative but not commutative, distributive through addition, contains an identity, and for each nonzero vector in four dimensions has a unique inverse [5] The quaternionic imaginary units $e_{i},(i, j, k)=(1,2,3)$ satisfy the relation:

$$
e_{i} e_{j}=-\delta_{i j}+\varepsilon_{i j k} e_{k}
$$

where $\varepsilon_{i j k}$ is the Levi-Civita symbol that is completely antisymmetric in the indices.

A real quaternion $q$ can be written in general as

$$
q=q_{0}+q_{1} e_{1}+q_{2} e_{2}+q_{3} e_{3}
$$

with $q_{0}, q_{1}, q_{2}, q_{3} \in \mathbb{R}, \mathbb{R}$ being the set of real numbers; $q_{0}$ is called the scalar part and $\left(q_{1} e_{1}+q_{2} e_{2}+q_{3} e_{3}\right)$ is the vector part of a quaternion. The conjugate of a quaternion is defined as

$$
\bar{q}=q_{0}-q_{1} e_{1}-q_{2} e_{2}-q_{3} e_{3}
$$

The norm of unit quaternion $q$ is $|q|=\sqrt{q \bar{q}}=1$ and $q^{-1}=\bar{q}$.

The scalar product of two arbitrary quaternions $p$ and $q$ is defined as

$$
(p, q)=\frac{1}{2}(\bar{p} q+\bar{q} p)=\frac{1}{2}(p \bar{q}+q \bar{p})
$$

The transformations of an arbitrary quaternion $t \rightarrow p t q$ and $t \rightarrow p \bar{t} q$ define orthogonal transformation of the group $O(4)$. It is clear that the above transformations preserve the norm $t \bar{t}=t_{0}^{2}+t_{1}^{2}+t_{2}^{2}+t_{3}^{2}$. We define the above transformations as abstract group operations by the notations

$$
t \rightarrow p t q:=[p, q] t, t \rightarrow p \bar{t} q:=[p, q]^{*} t .
$$

Dropping also $t$, the pair of quaternions define a set closed under multiplication.

The inverse elements take the forms [6]:

$$
[p, q]^{-1}=[\bar{p}, \overline{\mathrm{q}}],\left([p, q]^{*}\right)^{-1}=[\bar{q}, \bar{p}]^{*} .
$$

With the choice of $q=\bar{p}$, the orthogonal transformations define a three parameter subgroup $O(3)$. The transformation $[p, \bar{p}]$ and $[p, \bar{p}]^{*}$ leaves the $S c(t)=t_{0}$ invariant. Therefore in 3D Euclidean space one can assume that the quaternions consist of only vector components, namely $t=t_{1} e_{1}+t_{2} e_{2}+t_{3} e_{3}$, satisfying $\bar{t}=-t$. With this restriction to the $3 \mathrm{D}$ space the element $[p, \bar{p}]^{*}$ takes a simpler form $[p, \bar{p}]^{*} \Rightarrow[p,-\bar{p}]$. Therefore the transformations of the group $O(3)$ can be written as $[p, \pm \bar{p}]$ where the sandwiching operator $[p, \bar{p}]$ represents the rotations around the vector $\operatorname{Vec}(p)=\left(p_{1}, p_{2}, p_{3}\right)$ and $[p,-\bar{p}]$ is a rotary inversion [1].

Reflection of a vector $\Lambda$ represented as a quaternion with respect to a plane orthogonal to the unit pure quaternion $q$ can be written as

$$
\Lambda \rightarrow-q \bar{\Lambda} q
$$

If we apply another reflection to the vector $\Lambda$ it will lead to a rotation. The product of two reflections is a rotation. We will display some of the finite subgroups of quaternions related to the tetrahedral and octahedral groups.

The set $T$ is given by the group elements

$$
T=\left\{ \pm 1, \pm e_{1}, \pm e_{2}, \pm e_{3}, \frac{1}{2}\left( \pm 1 \pm e_{1} \pm e_{2} \pm e_{3}\right)\right\}
$$

and is called the binary tetrahedral group of order 24 . Another set of 24 quaternions is defined by

$$
T^{\prime}=\left\{\begin{array}{l}
\frac{1}{\sqrt{2}}\left( \pm 1 \pm e_{1}\right), \frac{1}{\sqrt{2}}\left( \pm 1 \pm e_{2}\right), \frac{1}{\sqrt{2}}\left( \pm 1 \pm e_{3}\right), \\
\frac{1}{\sqrt{2}}\left( \pm e_{1} \pm e_{2}\right), \frac{1}{\sqrt{2}}\left( \pm e_{2} \pm e_{3}\right), \frac{1}{\sqrt{2}}\left( \pm e_{3} \pm e_{1}\right)
\end{array}\right\}
$$




\section{SYMMETRY OF PYRITOHEDRON AND LATTICES}

The set $O=T \cup T^{\prime}$ forms binary octahedral group of order 48. These sets play an important role in the definition of the Pyritohedral subgroup.

\section{Finite Coxeter Groups, Cartan Matrix and Root Systems}

In 1934 Coxeter classified all finite Euclidean reflection groups [7-8]. A Coxeter group is a group $W(G)$ which has a presentation with a very special form

$$
W(G)=\left\langle r_{1}, r_{2}, \ldots, r_{n} \mid\left(r_{i} r_{j}\right)^{m_{i j}}=1\right\rangle
$$

where $r_{1}, r_{2}, \ldots, r_{n}$ are the reflection generators of the group.

All information of a root system can be encoded by Coxeter-Dynkin diagrams and the Cartan matrix $C$. The simple roots $\alpha_{1}, \alpha_{2}, \ldots, \alpha_{n}$ of the Coxeter-Dynkin diagram are the vectors orthogonal to certain hyperplanes with respect to which the simple reflection acts as an arbitrary vector $\lambda$ as [1]:

$$
r_{i} \lambda=\lambda-\frac{2\left(\lambda, \alpha_{i}\right)}{\left(\alpha_{i}, \alpha_{i}\right)} \alpha_{i}
$$

The Cartan matrix is a square integer matrix that links the simple roots of a given group through the following relation:

$$
C_{i j}=\frac{2\left(\alpha_{i}, \alpha_{j}\right)}{\left(\alpha_{j}, \alpha_{j}\right)}
$$

The inverse of the Cartan matrix is related to the metric of the dual (reciprocal) space as:

$$
G_{i j}=\left(C^{-1}\right)_{i j} \frac{\left(\alpha_{j}, \alpha_{j}\right)}{2}
$$

The basis vectors in the dual space are called the weight vectors, denoted by $\omega_{i}$, satisfying the scalar product: $G_{i j}=$ $\left(\omega_{i}, \omega_{j}\right)$. The simple roots and weight vectors are related to each other by

$$
\left(\omega_{i}, \frac{2 \alpha_{j}}{\left(\alpha_{j}, \alpha_{j}\right)}\right)=\delta_{i j} \text { and } \alpha_{i}=C_{i j} \omega_{j}
$$

\subsection{Coxeter-Dynkin diagram of $W\left(D_{3}\right)$}

The Coxeter-Dynkin diagram of $D_{3}$ with the quaternionic simple roots is shown in Figure 1 . The angle between two connected roots is $120^{\circ}$, otherwise they are orthogonal.

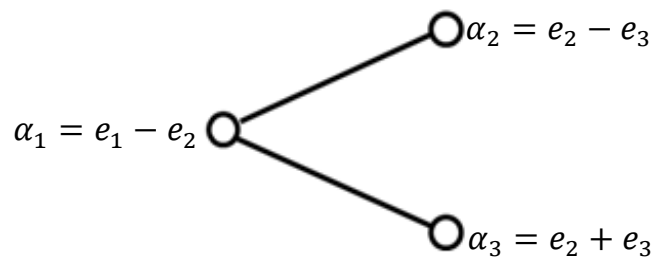

Figure 1. The Coxeter-Dynkin diagram $\boldsymbol{D}_{\mathbf{3}}$ with quaternionic simple roots.

An arbitrary quaternion $\lambda$ when reflected by the operator $r_{i}$ with respect to the hyperplane orthogonal to the quaternion $\alpha_{i}$, is given in terms of quaternion multiplication [6] as

$$
r_{i} \lambda=-\frac{\alpha_{i}}{\sqrt{2}} \bar{\lambda} \frac{\bar{\alpha}_{i}}{\sqrt{2}}:=\left[\frac{\alpha_{i}}{\sqrt{2}},-\frac{\alpha_{i}}{\sqrt{2}}\right]^{*} \lambda:=\left[\frac{\alpha_{i}}{\sqrt{2}}, \frac{\alpha_{i}}{\sqrt{2}}\right], i=1,2,3 .
$$

The generators of the Coxeter group $W\left(D_{3}\right)$ are then given in the notation of (14) by

$$
\begin{gathered}
r_{1}=\left[\frac{1}{\sqrt{2}}\left(e_{1}-e_{2}\right),-\frac{1}{\sqrt{2}}\left(e_{1}-e_{2}\right)\right]^{*}=\left[\frac{1}{\sqrt{2}}\left(e_{1}-e_{2}\right), \frac{1}{\sqrt{2}}\left(e_{1}-e_{2}\right)\right], \\
r_{2}=\left[\frac{1}{\sqrt{2}}\left(e_{2}-e_{3}\right),-\frac{1}{\sqrt{2}}\left(e_{2}-e_{3}\right)\right]^{*}=\left[\frac{1}{\sqrt{2}}\left(e_{2}-e_{3}\right), \frac{1}{\sqrt{2}}\left(e_{2}-e_{3}\right)\right], \\
r_{3}=\left[\frac{1}{\sqrt{2}}\left(e_{2}+e_{3}\right),-\frac{1}{\sqrt{2}}\left(e_{2}+e_{3}\right)\right]^{*}=\left[\frac{1}{\sqrt{2}}\left(e_{2}+e_{3}\right), \frac{1}{\sqrt{2}}\left(e_{2}+e_{3}\right)\right] .
\end{gathered}
$$


They generate the Coxeter-Weyl group $W\left(D_{3}\right)$ of order 24, isomorphic to the tetrahedral group, the elements of which can be written compactly by the notation

$$
W\left(D_{3}\right)=\left\{[T, \bar{T}] \cup\left[T^{\prime},-\bar{T}^{\prime}\right]\right\} .
$$

Here $T$ and $T^{\prime}$ are the sets of quaternions given in (8-9). When the simple roots are chosen as in Figure 1, then the weight vectors are determined as

$$
\begin{aligned}
& \omega_{1} \equiv(100)=e_{1}, \\
& \omega_{2} \equiv(010)=\frac{1}{2}\left(e_{1}+e_{2}-e_{3}\right), \\
& \omega_{3} \equiv(001)=\frac{1}{2}\left(e_{1}+e_{2}+e_{3}\right) .
\end{aligned}
$$

Using the orbit definition $W\left(D_{3}\right)\left(a_{1} a_{2} a_{3}\right):=\left(a_{1} a_{2} a_{3}\right)_{D_{3}}$ all the orbits can be determined.

\subsection{Coxeter-Dynkin diagram $W\left(D_{3}\right)$ with Dynkin diagram symmetry}

The symmetry of the union of the orbits $(010)_{D_{3}} \cup(001)_{D_{3}}$ requires the Dynkin- diagram symmetry : $\alpha_{1} \rightarrow \alpha_{1}, \alpha_{2} \leftrightarrow$ $\alpha_{3}$ as shown in Figure 2. It leads to the transformation on the imaginary quaternions $\gamma: e_{1} \rightarrow e_{1}, e_{2} \rightarrow e_{2}, e_{3} \rightarrow-e_{3}$. The Dynkin-diagram symmetry operator reads $\gamma=\left[e_{3},-e_{3}\right]^{*}=\left[e_{3}, e_{3}\right]$ which extends the Coxeter group $W\left(D_{3}\right)$ to the octahedral group $\operatorname{Aut}\left(D_{3}\right) \approx W\left(D_{3}\right): C_{2}$ of order 48, the automorphism group of the root system of $D_{3}$.

$$
O_{h} \approx \operatorname{Aut}\left(D_{3}\right)=\left\{[T, \pm \bar{T}] \cup\left[T^{\prime}, \pm \bar{T}^{\prime}\right]\right\} \text {. }
$$

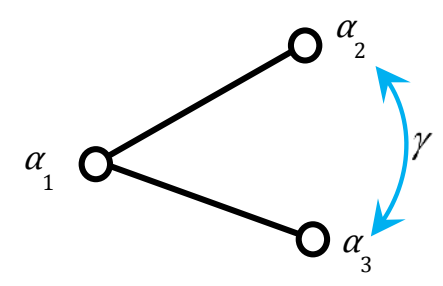

Figure 2. The Dynkin-diagram symmetry $\gamma$.

The maximal subgroups of the octahedral group $\operatorname{Aut}\left(D_{3}\right) \approx O_{h}$, each of order 24 , are shown in Figure 3 . In the next section we work on pyritohedral group.

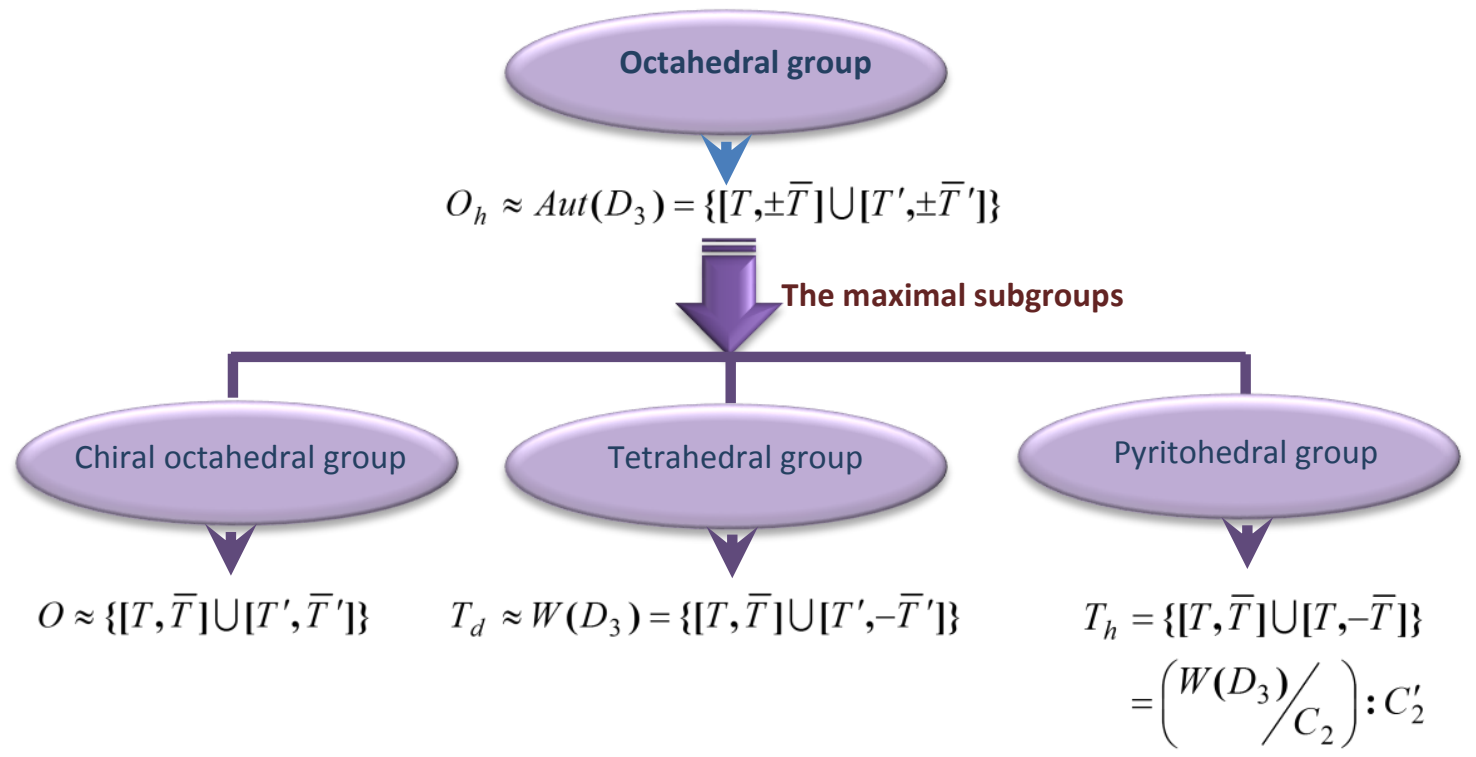

Figure 3. The maximal subgroups of the octahedral group. 


\section{SYMMETRY OF PYRITOHEDRON AND LATTICES}

\section{Pyritohedral Group and Related Polyhedra}

Pyritohedral symmetry is well known in crystallography as the symmetry of the pyritohedron. The group of this symmetry is an important discrete point group of crystallography. The polyhedra with this symmetry can be derived from the Coxeter-Dynkin diagram of $D_{3}$. The pyritohedral group $T_{h}=\{[T, \bar{T}] \cup[T,-\bar{T}]\}$ consists of rotation generators in addition to the Dynkin diagram symmetry $\gamma$ of $D_{3}$. The group can be represented as $T_{h}=A_{4}: C_{2}=\left\langle r_{1} r_{2}, r_{1} r_{3}, \gamma\right\rangle$ where $(:)$ denotes the semi direct product. This is the rotational symmetry of a cube with stripes on its faces as shown in Figure 4. The rotation generators $r_{1} r_{2}$ and $r_{1} r_{3}$ of $D_{3}$ generate the subgroup $A_{4} \approx W\left(D_{3}\right) / C_{2}=[T, \bar{T}]$ of order 12 , where $A_{4}$ is an even permutation of 4 vertices of a tetrahedron. The chiral tetrahedral group $[T, \bar{T}] \approx A_{4}$ consists of 8 rotations by $120^{\circ}$ around the 4 diagonals of the cube, 3 rotations by $180^{\circ}$ around the $x, y$ and $z$ axes, and the unit element [9]. Pyrite crystals often occur in the forms of cubes with striated faces as in Figure 4, octahedra and pyritohedra (a solid similar to dodecahedron but with non-regular pentagonal faces) or some combinations of these forms.

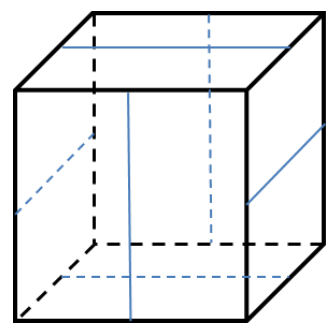

Figure 4. The cube with stripes on its faces possesses the pyritohedral symmetry.

\subsection{Construction of the vertices of the pseudoicosahedron}

We denote a general vector of $D_{3}$ by $\lambda=a_{1} \omega_{1}+a_{2} \omega_{2}+a_{3} \omega_{3}$ where the weight vectors are defined in (17). We note that the orbit of the pyritohedral group generated by the vector $\lambda$ with different values of $a_{i}$ forms different polyhedra. For instance:

$a_{1}=1, a_{2}=a_{3}=0$ corresponds to an octahedron.

$a_{1}=a_{3}=0, a_{2}=1$ or $a_{1}=a_{2}=0, a_{3}=1$ corresponds to a cube.

$a_{1}=0, a_{2}=a_{3}=1$ corresponds to a cuboctahedron.

$a_{1} \neq 0, a_{2}=a_{3} \neq 0$ gives $\lambda=a_{1}\left(e_{1}+\frac{a_{2}}{a_{1}}\left(e_{1}+e_{2}\right)\right)$, and the orbit corresponds to a pseudoicosahedron as discussed below.

Applying some of the rotation elements of the pyritohedral symmetry on a general vector of $\lambda=a_{1} \omega_{1}+a_{2} \omega_{2}+a_{3} \omega_{3}$, one can generate five triangles sharing the vertex $\lambda$ as shown in Figure 5(a), which consist of two equilateral and three isosceles triangles. The vertices $\lambda, r_{1} r_{2} \lambda$ and $\left(r_{1} r_{2}\right)^{2} \lambda$ form an equilateral triangle with an edge length squared: $a_{1}^{2}+a_{1} a_{2}+a_{2}^{2}$. Similarly, the vertices $\lambda, r_{1} r_{3} \lambda$ and $\left(r_{1} r_{3}\right)^{2} \lambda$ form the second equilateral triangle with the edge length squared: $a_{1}^{2}+a_{1} a_{3}+a_{3}^{2}$. The line between the vertices $\lambda$ and $r_{2} r_{3} \lambda$ has a length squared: $a_{2}^{2}+a_{3}^{2}$. If we impose all the edge lengths to be equal, we would have five equilateral triangles around one vertex and obtain the relation 


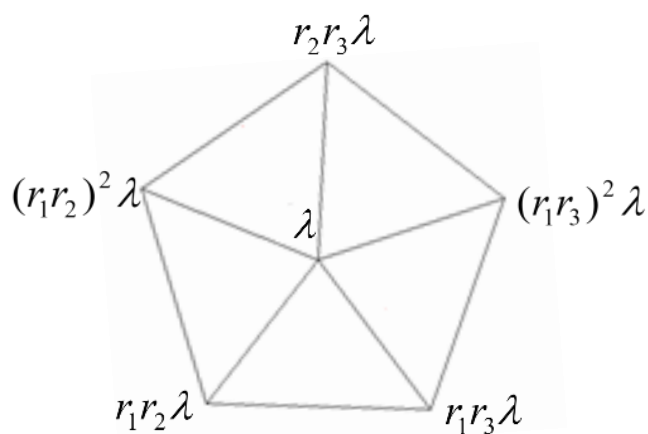

(a)

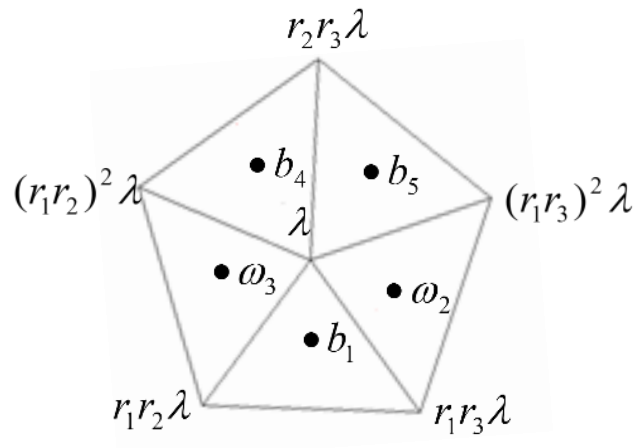

(b)

Figure 5. (a) Five triangles meeting at one vertex, (b) The normal vectors of the triangles surrounding $\lambda$.

$$
a_{1}^{2}+a_{1} a_{2}+a_{2}^{2}=a_{1}^{2}+a_{1} a_{3}+a_{3}^{2}=a_{2}^{2}+a_{3}^{2}
$$

Suppose the Dynkin-diagram symmetry as shown diagrammatically in Figure 2 leaves the general vector $\lambda$ invariant $\gamma \lambda=\lambda$. This symmetry exchanges $\omega_{2}$ and $\omega_{3}$, so that

$$
\gamma \lambda=\lambda \Rightarrow a_{1} \omega_{1}+a_{2} \omega_{3}+a_{3} \omega_{2}=a_{1} \omega_{1}+a_{2} \omega_{2}+a_{3} \omega_{3}
$$

From (19) one gets $a_{2}=a_{3}$, and a general vector can be written in terms of quaternions as

$$
\lambda=a_{1}\left(\omega_{1}+x\left(\omega_{2}+\omega_{3}\right)\right)=a_{1}\left((1+x) e_{1}+x e_{2}\right)
$$

where $x=\frac{a_{2}}{a_{1}}=\frac{a_{3}}{a_{1}}$ is a parameter which could be computed from (19). If we take a general value for $x$ in the vector given in (21), the action of the pyritohedral group on $\lambda$ will generate the set of 12 vectors (apart from the scale factor $\left.a_{1}\right)$ :

$$
T_{h} \lambda= \pm(1+x) e_{1} \pm x e_{2}, \pm(1+x) e_{2} \pm x e_{3}, \pm(1+x) e_{3} \pm x e_{1}
$$

Factoring (19) by $a_{1}$ one obtains the equation $x^{2}-x-1=0$. The solutions of this equation are $\tau=\frac{1+\sqrt{5}}{2}$ and $\sigma=\frac{1-\sqrt{5}}{2}$. These values of $x$ will lead to five equilateral triangles sharing the vertex $\lambda$. Substituting $\tau$ and $\sigma$ respectively for $x$ in (22) we obtain two sets of 12 vertices as:

$$
\begin{gathered}
\tau\left\{ \pm \tau e_{1} \pm e_{2}, \pm \tau e_{2} \pm e_{3}, \pm \tau e_{3} \pm e_{1}\right\} \\
\sigma\left\{ \pm \sigma e_{1} \pm e_{2}, \pm \sigma e_{2} \pm e_{3}, \pm \sigma e_{3} \pm e_{1}\right\}
\end{gathered}
$$

These sets of vertices represent two mirror images of an icosahedron with a scale factor difference. Multiplying the vertices in (23b) by $\tau^{3}$ one obtains the following set of quaternions:

$$
\tau\left\{ \pm e_{1} \pm \tau e_{2}, \pm e_{2} \pm \tau e_{3}, \pm e_{3} \pm \tau e_{1}\right\}
$$

The vectors here have the same norm as in (23a). The icosahedron represented by (23a) is shown in Figure 6(a). Table 1 summarizes the action of the pyritohedral group on $\lambda$ for various values of $x$ : 
Table 1. The action of the pyritohedral group for various values of $x$.

\begin{tabular}{|c|c|c|c|}
\hline & $\lambda=(1+x) e_{1}+x e_{2}$ & $T_{h} \lambda$ & $\begin{array}{c}\text { Polyhedra } \\
\text { generated }\end{array}$ \\
\hline$x=\tau$ & $\lambda=\tau\left(\tau e_{1}+e_{2}\right)$ & $\tau\left\{ \pm \tau e_{1} \pm e_{2}, \pm \tau e_{2} \pm e_{3}, \pm \tau e_{3} \pm e_{1}\right\}$ & Icosahedron \\
or $x=\sigma$ & or & or & Octahedron \\
\hline$x=0$ or & $\lambda=\sigma\left(\sigma e_{1}+e_{2}\right)$ & $\sigma\left\{\sigma e_{1} \pm e_{2}, \pm \sigma e_{2} \pm e_{3}, \pm \sigma e_{3} \pm e_{1}\right\}$ & \\
$x=-1$ & $\lambda=e_{1}$ & $\pm e_{1}, \pm e_{2}, \pm e_{3}$ & \\
\hline or & $\lambda=-e_{2}$ & $\pm e_{1} \pm e_{2}, e_{2} \pm e_{3}, \pm e_{3} \pm e_{1}$ & Cuboctahedron \\
\hline$x=-\frac{1}{2}$ & $\lambda=\frac{1}{2}\left(e_{1}-e_{2}\right)$ & $\pm(1+x) e_{1} \pm x e_{2}, \pm(1+x) e_{2} \pm x e_{3}, \pm(1+x) e_{3} \pm x e_{1}$ & Pseudoicosahedron \\
\hline Other $x$ & $\lambda=(1+x) e_{1}+x e_{2}$ & & \\
\hline
\end{tabular}

The vertices of the dual of the icosahedron, say the set of vectors of (23a), can be determined as [9]:

$$
\begin{gathered}
\frac{1}{2}\left\{ \pm \sigma e_{1} \pm \tau e_{2}, \pm \sigma e_{2} \pm \tau e_{3}, \pm \sigma e_{3} \pm \tau e_{1}\right\} \\
\frac{1}{2}\left( \pm e_{1} \pm e_{2} \pm e_{3}\right) .
\end{gathered}
$$

The 20 vertices in (25a and 25b) represent a dodecahedron as shown in Figure 6 (b). Its mirror image can be obtained by replacing $\sigma \leftrightarrow \tau$.

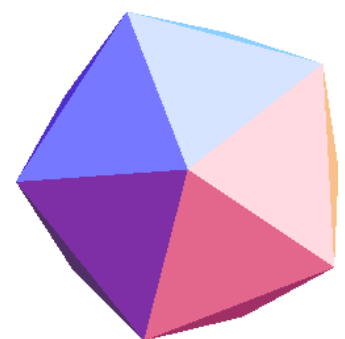

(a)

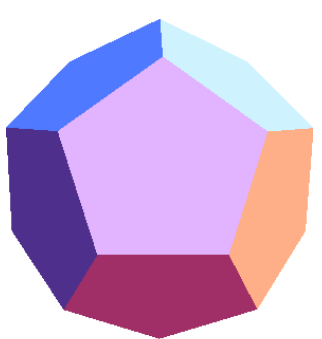

(b)

Figure 6. (a) Icosahedron, (b) dodecahedron (dual of the icosahedron).

The vectors in $(25 b)$ represent the vertices of a cube which is invariant under the pyritohedral symmetry. Similarly, the other 12 vertices of (25a) form another orbit under the pyritohedral symmetry. They are obtained for $x=-\tau$ in $(22)$ leading to a pseudoicosahedron. 


\subsection{Construction of the vertices of the pyritohedron from the pseudoicosahedron}

We can compute the dual of the pseudoicosahedron in equation (22) for an arbitrary value of $x$. This can be achieved by determining the vectors normal to the faces of the pseudoicosahedron in (22). The normal vectors of the five triangles in Figure 5 (b) can be determined as follows:

The normal vector of the triangle with the vertices $\lambda, r_{1} r_{2} \lambda,\left(r_{1} r_{2}\right)^{2} \lambda$ can be taken as $\omega_{3}$ because $r_{1} r_{2}$ is a rotation around $\omega_{3}$ for $r_{1} r_{2} \omega_{3}=\omega_{3}$.

Similarly, the normal vector of the triangle with the vertices $\lambda, r_{1} r_{3} \lambda$ and $\left(r_{1} r_{3}\right)^{2} \lambda$ can be taken as $\omega_{2}$ that is $r_{1} r_{3} \omega_{2}=\omega_{2}$. The vertices generated by the pyritohedral group from either $\omega_{2}$ or $\omega_{3}$ would lead to the vertices of a cube given in (25b).

The vectors normal to the isosceles triangles shown in Figure 5 (b) can be computed as follows:

$$
\begin{aligned}
& b_{1}=e_{1}+(1+x) e_{2}, \\
& b_{4}=(1+x) e_{1}+e_{3}, \\
& b_{5}=(1+x) e_{1}-e_{3} .
\end{aligned}
$$

The vectors $\omega_{2}$ and $\omega_{3}$ are in the same orbit under the pyritohedral group. Moreover, $\left(\omega_{3}-\omega_{2}\right)$ is orthogonal to $\lambda=(1+x) e_{1}+x e_{2}$ which means the scalar product of $\left(\omega_{3}-\omega_{2}\right)$ and $\lambda$ is zero: $\left(\left(\omega_{3}-\omega_{2}\right), \lambda\right)=0$. On the other hand, one can prove that $b_{1}, b_{4}$ and $b_{5}$ are also in one orbit under the pyritohedral group. They form a plane orthogonal to the vector $\lambda=(1+x) e_{1}+x e_{2}$. As these five vertices should determine the same plane then one can show that $\left(\left(\rho b_{1}-\omega_{2}\right), \lambda\right)=0$ is the necessary condition to determine the scale factor $\rho$. From this relation we obtain $\rho=$ $\frac{1+2 x}{2(1+x)^{2}}$ where the scale factor implies that $x \neq 1$ and $x \neq-\frac{1}{2}$.

Then the vectors $\rho b_{1}, \rho b_{4}, \rho b_{5}, \omega_{2}$ and $\omega_{3}$ determine a pentagon, non-regular in general (four edges of the same length and one edge different), as shown in Figure 7.

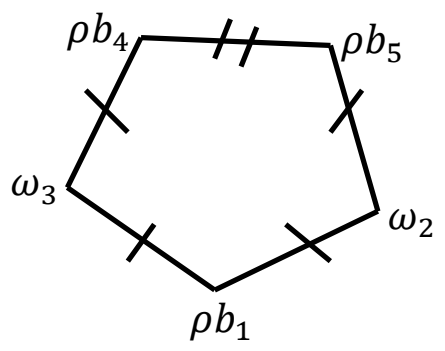

Figure 7. The plane determined by the vectors normal to the faces of the pseudoicosahedron in equation (22).

Applying the pyritohedral group on these 5 vertices we obtain 20 vectors where $h=x /(x+1)$. The following 20 vertices of the pyritohedron split into two orbits: 12 vertices form a pseudoicosahedron and 8 vertices $\left( \pm e_{1} \pm e_{2} \pm e_{3}\right)$ form a cube .

$$
\begin{gathered}
\left\{ \pm\left(1-h^{2}\right) e_{1} \pm(1+h) e_{2}, \pm\left(1-h^{2}\right) e_{2} \pm(1+h) e_{3}, \pm\left(1-h^{2}\right) e_{3} \pm(1+h) e_{1}\right\} \\
\left( \pm e_{1} \pm e_{2} \pm e_{3}\right)
\end{gathered}
$$

The pyritohedron has a geometric degree of freedom with two limiting cases as shown in Figure 8. 

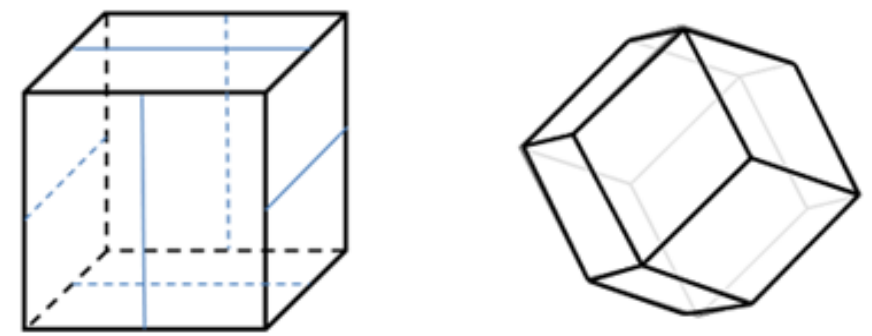

Figure 8. Special cases of pyritohedron for (a) $h=0$, (b) $h=1$.

When $h=0$, the $12+8$ vertices in (27) represent the centers of edges and the vertices of a cube as in Figure 8 (a). When $h=1$, the vertices in (27) represent a rhombic dodecahedron as shown in Figure 8 (b). The regular dodecahedron represents a special intermediate case $(x=-\sigma$ or $x=-\tau)$ where all edges and angles are equal.

\section{Vertices of the Pseudoicosahedron and the Pyritohedron in a Lattice}

The simple cubic lattice consists of the vector $m_{1} e_{1}+m_{2} e_{2}+m_{3} e_{3}$ where $m_{i} \in \boldsymbol{Z}, i=1,2,3$. Now, we can discuss the pseudoicosahedron and its dual pyritohedron relevant to crystallography. Many candidates of pseudoicosahedra and its dual pyritohedra can be obtained as a structure in the simple cubic lattice. The vertices of pseudoicosahedron and its dual pyritohedron for various $x$ (and corresponding $h$ ) values are given in Table 2 and are plotted in Figure 9. As mentioned in Section 4.2, when $x \rightarrow 0 \quad(h \rightarrow 0)$ the pseudoicosahedron is converted to an octahedron and the pyritohedron becomes a cube. The set of vertices of the pseudoicosahedron and the pyritohedron belong to the simple cubic lattice.

The unit cubic cell can be stacked in the pseudoicosahedron or in the pyritohedron as long as the vertices are chosen from the simple cubic lattice.

Table 2. Pseudoicosahedron and its dual (pyritohedron) for arbitrary $x$ values.

\begin{tabular}{|c|c|c|c|}
\hline$x$ & $h$ & Vertices of pseudoicosahedron & $\begin{array}{l}\text { Vertices of pyritohedron } \\
\text { (up to a scale factor) }\end{array}$ \\
\hline$\frac{5}{3}$ & $\frac{5}{8}$ & $\begin{array}{l}\left. \pm 8 e_{1} \pm 5 e_{2}, \pm 8 e_{2} \pm 5 e_{3}, \pm 8 e_{3} \pm 5 e_{1}\right) \\
\text { For } a_{1}=3 \text { with faces isosceles triangles with } \\
\text { edge ratios } 1,1, \sqrt{50 / 49} .\end{array}$ & $\begin{array}{c}\left\{ \pm 39 e_{1} \pm 104 e_{2}, \pm 39 e_{2}\right. \\
\pm 104 e_{3}, \pm 39 e_{3} \\
\left. \pm 104 e_{1}\right\} \\
64\left( \pm e_{1} \pm e_{2} \pm e_{3}\right)\end{array}$ \\
\hline 1 & $\frac{1}{2}$ & $\begin{array}{l}\quad\left( \pm 2 e_{1} \pm e_{2}, \pm 2 e_{2} \pm e_{3}, \pm 2 e_{3} \pm e_{1}\right) \\
\text { with faces isosceles triangles of edge ratios } 1,1, \\
\sqrt{\frac{2}{3}}\end{array}$ & $\begin{array}{c}\left\{ \pm 3 e_{1} \pm 6 e_{2}, \pm 3 e_{2} \pm 6 e_{3}, 3 e_{3}\right. \\
\left. \pm 6 e_{1}\right\} \\
4\left( \pm e_{1} \pm e_{2} \pm e_{3}\right)\end{array}$ \\
\hline$\frac{1}{10}$ & $\frac{1}{11}$ & $\begin{array}{l}\left( \pm 11 e_{1} \pm e_{2}, \pm 11 e_{2} \pm e_{3}, \pm 11 e_{3} \pm e_{1}\right) \\
\text { For } a_{1}=4, \text { faces of with isosceles triangles of } \\
\text { edges } 1,1, \sqrt{2 / 111}\end{array}$ & $\begin{array}{l}\left\{ \pm 110 e_{1} \pm 132 e_{2}, \pm 110 e_{2}\right. \\
\pm 132 e_{3}, \pm 110 e_{3} \\
\left. \pm 132 e_{1}\right\}, 111\left( \pm e_{1} \pm e_{2} \pm e_{3}\right)\end{array}$ \\
\hline
\end{tabular}




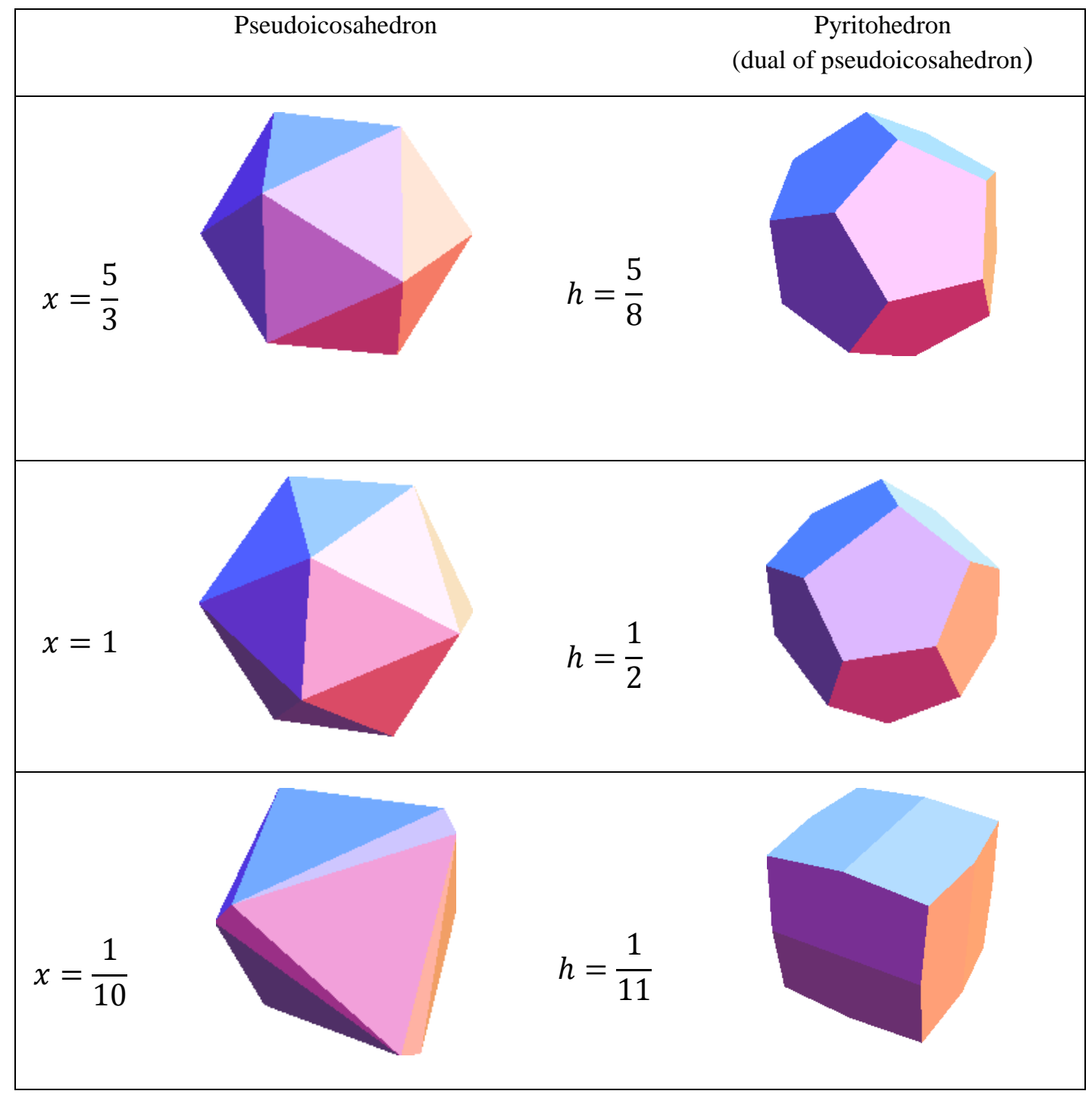

Figure 9. The pseudoicosahedron and its dual (pyritohedron) for various $x$ values.

For a general $x$, the union of two pseudoicosahedra

$$
\begin{aligned}
& \pm(1+x) e_{1} \pm x e_{2}, \pm(1+x) e_{2} \pm x e_{3}, \pm(1+x) e_{3} \pm x e_{1} \\
& \pm x e_{1} \pm(1+x) e_{2}, \pm x e_{2} \pm(1+x) e_{3}, \pm x e_{3} \pm(1+x) e_{1}
\end{aligned}
$$

represents the vertices of a non-regular truncated octahedron which can be derived as the orbit of the Coxeter group $W\left(B_{3}\right)$ denoted by $a_{1}(1, x, 0)_{B_{3}}$. This belongs to the simple cubic lattice if $a_{1}$ and $a_{1} x=a_{2}$ are integers, which implies that $x$ should be a rational number. In other words, as long as $a_{1}$ and $a_{2}$ are integers the pseudoicosahedron with the vertices can be embedded in a simple cubic lattice.

\section{Conclusion}

$$
\pm\left(a_{1}+a_{2}\right) e_{1} \pm a_{2} e_{2}, \pm\left(a_{1}+a_{2}\right) e_{2} \pm a_{2} e_{3}, \pm\left(a_{1}+a_{2}\right) e_{3} \pm a_{2} e_{1}
$$

The polyhedra possessing the pyritohedral symmetry have been constructed in terms of quaternions. Crystals with pyritohedral symmetry exist in the form of a stratified cube, an octahedron and a pyritohedron. It is expected that crystal structures in the form of a pseudoicosahedron as well as a pseudoicosidodecahedron may exist.

The relevance of the pseudoicosahedron to pyritohedral crystals could stimulate research in application to material science. Representation of the pyritohedral symmetry and crystal vectors in term of quaternions is more demanding, but may lead to a new understanding of crystal structures and their symmetries. We are anticipating that this finding will form a link between quaternions and crystallography. 


\section{SYMMETRY OF PYRITOHEDRON AND LATTICES}

\section{References}

1. Coxeter, H.S.M. Regular polytopes ( $3^{\text {rd }}$ ed.) New York: Dover Publications, 1973.

2. Koca, M., Al-Ajmi, M. and Koc, R. Polyhedra obtained from Coxeter groups and quaternions, J. Math. Phys, 2007, 48, 113514-113527.

3. Koca, M., Koca, N.O. and Koc, R. Catalan solids derived from three dimensional root systems and quaternions, $J$. Math. Phys, 2010, 51(4), 043501-043513.

4. Conway, J.H. and Smith D.A. On quaternions and octonions: Their geometry, Aritmetic and symmetry, A.K. Peters, Ltd. Natick, M.A., 2003.

5. Hamilton, S.W.R. Lectures on Quaternions, London: Whittaker, 1853.

6. Koca, M., Koc, R. and Al-Barwani, M. Non-crystallographic Coxeter group $H_{4}$ in E8. J. Phys. A: Math. Gen. A, 2001, 34,11201-11213.

7. Coxeter, H.S.M. Discrete groups generated by reflections. Annals of Mathematics, 1946, 35, 588-621.

8. Steinberg, R. Finite reflection grouups. Trans. Am. Math. Soc., 1959, 493-504.

9. Koca, M., Koca, N.O. and Al-Shu'eili, M. Chiral Polyhedra derived from Coxeter diagrams and quaternions, $S Q U J$. Sci., 2011, 1, 16, 63; arXiv: 1006.3149.

Received 7 June 2016

Accepted 4 September 2016 